\title{
Application of an Analytical Method to Locate a Mixing Plane in a Supersonic Compressor
}

\author{
Emmanuel Benichou ${ }^{1}$ and Isabelle Trébinjac ${ }^{2}$ \\ 1. Turbomeca, Groupe SAFRAN, Bordes 64511, France \\ 2. Laboratoire de Mécanique des Fluides et d'Acoustique, UMR CNRS 5509, Ecole Centrale de Lyon, Ecully Cedex 69134, France
}

Received: September 12, 2014 / Accepted: November 03, 2014 / Published: January 31, 2015.

\begin{abstract}
In order to achieve greater pressure ratios, compressor designers have the opportunity to use transonic configurations. In the supersonic part of the incoming flow, shock waves appear in the front part of the blades and propagate in the upstream direction. In case of multiple blade rows, steady simulations have to impose an azimuthal averaging (mixing plane) which prevents these shock waves to extend upstream. In the present paper, several mixing plane locations are numerically tested and compared in a supersonic configuration. An analytical method is used to describe the shock pattern. It enables to take a critical look at the CFD (computational fluid dynamics) steady results. Based on this method, the shock losses are also evaluated. The good agreement between analytical and numerical values shows that this method can be useful to wisely forecast the mixing plane location and to evaluate the shift in performances due to the presence of the mixing plane.
\end{abstract}

Key words: Supersonic compressor, shock wave, pressure loss, RANS, mixing plane.

\section{Nomenclature}

\section{Symbol}

\section{$P$}

$P_{t}$

$\overline{P_{t}}$

$t$

$\rho$

$V$

$V_{n}$

$V_{t 1}, V_{t 2}$

$r$

$a$

$\gamma$

$\Omega$

$x_{0}$

$x, y$

$M$
Static pressure

Stagnation pressure in the impeller frame Circumferentially averaged stagnation pressure

Circumferential pitch

Density

Velocity in the impeller frame

Normal velocity component

Tangential velocity components

Radius

Speed of sound

Perfect gas constant

Rotation speed

Position of the bow shock on the profile symmetry axis

Coordinates of a point in the profile frame

Mach number

Corresponding author: Emmanuel Benichou, Ph.D. student, research fields: aerodynamic instabilities in centrifugal compressors, including rotor-stator interactions and flow control issues using boundary layer aspiration. E-mail: emmanuel.benichou@ec-lyon.fr.

Mach angle

Angle of the flow

Angle of the bow shock

Axial distance between points $x_{0}$ and $\mathrm{B}$

Detachment distance of the shock wave

Pitchwise distance on which the flow is considered isentropic

Circumferential direction

Total pressure loss coefficient

\section{Subscript}

B, C Relative to points B and C

$1,2 \quad$ Calculated in Section 1 or Section 2

$\infty \quad$ Value of the quantity at infinite upstream

\section{Exponent}

$$
\text { * } \quad \text { Value of the quantity at } M=1
$$

\section{Introduction}

The need for compact, efficient high pressure ratio compressors often results in high rotation speeds. In some cases, the entry flow may therefore be supersonic over the entire- or upper-span. The resulting physics of the flow field in the entry zone can be complex because 
of the interaction between compression and expansion waves [1-3]. Besides, shock waves propagating upstream the blades are dissipative and must necessarily be taken into account in the prediction of the stage performances [4].

Current CFD (computational fluid dynamics) offers three main categories for simulations: RANS (Reynolds-averaged Navier-Stokes), LES (large eddy simulation) and DNS (direct numerical simulation). RANS simulations approximate the mean effect of turbulence, while DNS enables a full resolution of the Navier-Stokes equations, from the smallest turbulence scale (Kolmogorov scale) up to the integral scale. LES corresponds to a filtered DNS: only the largest turbulence scales are resolved, the smallest ones being modeled. The more turbulence scales are resolved, the finer the mesh must be, and thus the more expensive the simulation becomes.

In a current engine design process, only RANS simulations can be carried out. This sort of simulation is based on the Reynolds decomposition and turbulence models are added to close the set of equations. U-RANS (unsteady RANS) simulations are generally not affordable in a conception approach, because of their high CPU cost. That is why the only tool typically available for designers today consists in steady RANS simulations. In the case of multi-row turbomachinery, these simulations rely most of the time on the use of mixing planes, which average the data in the circumferential direction, and thus do not let the non-uniformities in the flow field transmit in the upstream or downstream direction.

This article focuses on the entry zone of a supersonic compressor. The filtration of the shock pattern upstream the blades by the mixing plane raises the issue of the mixing plane location. The present paper compares numerically different locations to point out the influence of the mixing plane on the flow field, notably in terms of stagnation pressure change.

In a first part, the numerical results are qualitatively analyzed and the role of the mixing plane is highlighted.
An analytical model is then used to reproduce the shape of the shock waves at the leading edge of the blades. Finally, a method is given that enables to judge the reliability of steady simulations on supersonic configurations.

\section{Test Case and Numerical Procedure}

The test case is a centrifugal unshrouded impeller designed and built by Turbomeca. In the present study, only the front part of it is concerned. There is therefore no need giving the compressor geometry and performance in this paper. Only one operating point is examined, and it corresponds to the sonic blockage region. The flow, supersonic over $60 \%$ of the span, is examined at several section heights between $h_{1}$ and $h_{2}$ in Fig. 1.

A simulation performed without any mixing plane is used as reference (Fig. 2a). In this one, the inlet block is rotating with the impeller, and there is no particular interface. In order to evaluate the change in performance induced by the mixing plane approach, three different mixing plane locations are numerically tested (Fig. 2b). They are labeled "a", "b" and "c" in Fig. 1. In those three simulations, the inlet block is fixed, like a stator row and the periodicity enables to use a smaller domain since the flow upstream of the mixing plane is circumferentially uniform.

Computations were performed with the elsA software developed at ONERA [5]. The code is based on a cell-centered finite volume method and solves the

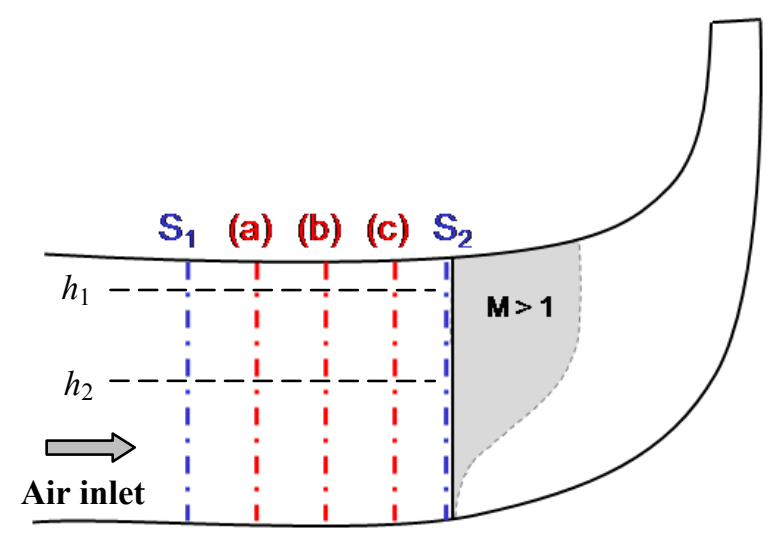

Fig. 1 Meridional sketch of the compressor inlet part. 


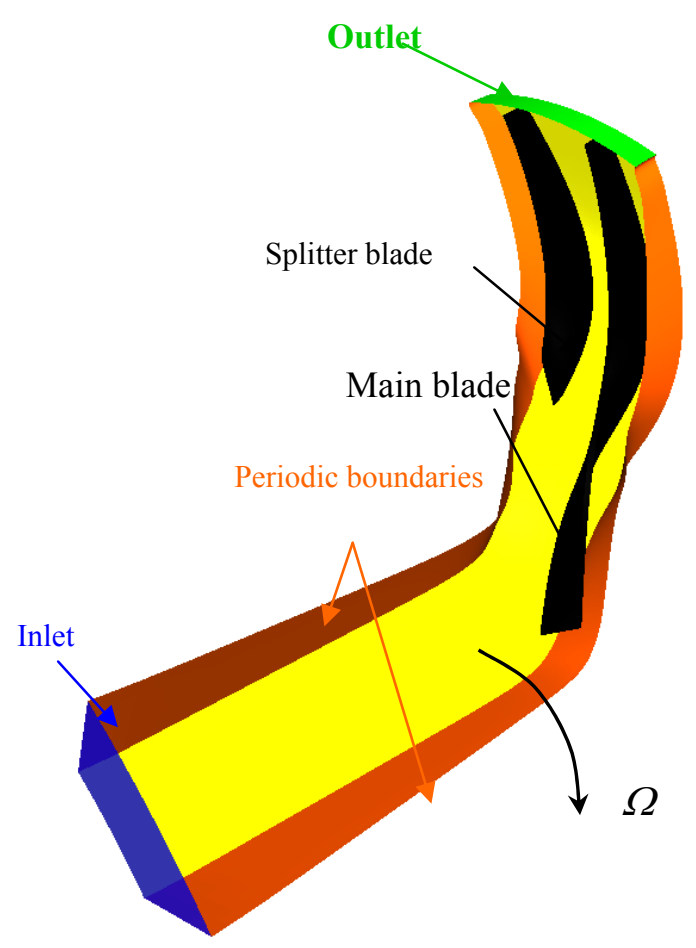

(a)

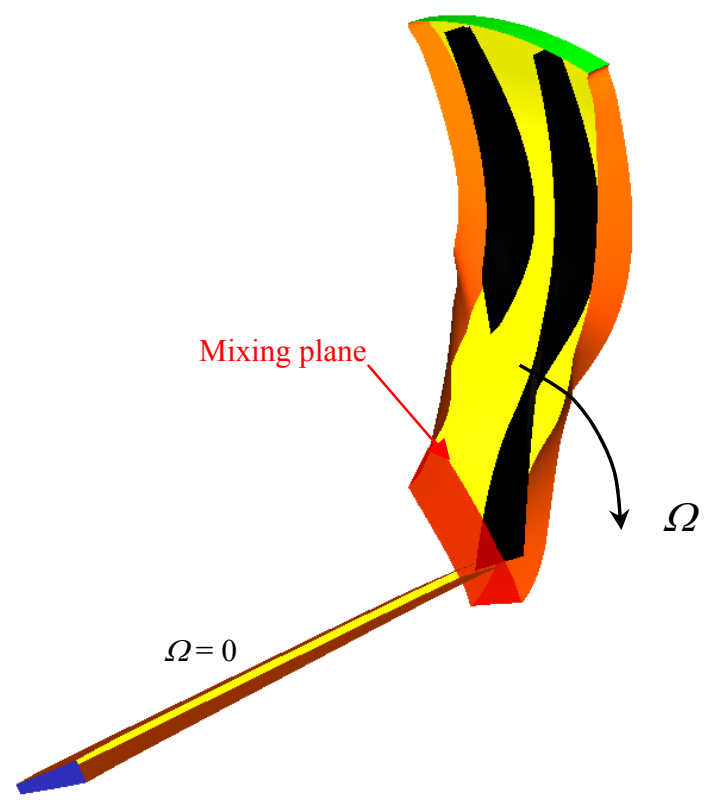

(b)

Fig. 2 (a) 3D view of the domain without mixing plane; and (b) 3D view of the domain with mixing plane.

compressible RANS equations on multi-block structured meshes. The $k-l$ model of Smith [6] (chosen according previous work [7]) is used for turbulence modeling. The set of equations are resolved in the relative frame of each row, using the Roe space scheme for convective fluxes and a 2 nd-order centered scheme for viscous fluxes. An LU implicit phase (lower upper decomposition) is associated to the backward-Euler scheme for time integration. The near-wall region is described with $\mathrm{y}^{+}<13$. The inlet condition imposes the velocity angles and the standard stagnation pressure and temperature. The turbulent values are determined from a free-stream turbulence rate of 5\% (resulting from previous measurements). The outlet condition imposes a uniform value of static pressure. The walls are described with non-slip and adiabatic conditions. The steady state enables to simulate only one blade passage, the azimuthal boundaries being periodic. Blade-to-blade surfaces are then extracted from all simulations at the same section heights between $h_{1}$ and $h_{2}$.

At the mixing plane interface, a circumferential average using Riemann invariants is computed at both upstream and downstream faces. The resulting values $\left(m_{1}-m_{5}\right)$ are then applied to the adjacent face with a non-reflective boundary condition:

$$
\left\{\begin{array}{l}
m_{1}=\frac{1}{S} \int\left(P-\rho a V_{n}\right) \mathrm{d} S \\
m_{2}=\frac{1}{S} \int\left(P+\rho a V_{n}\right) \mathrm{d} S \\
m_{3}=\frac{1}{S} \int\left(P-\rho a^{2}\right) \mathrm{d} S \\
m_{4}=\frac{1}{S} \int V_{t_{1}} \mathrm{~d} S \\
m_{5}=\frac{1}{S} \int V_{t_{2}} \mathrm{~d} S
\end{array}\right.
$$

with $S=\int \mathrm{d} S$ the total surface of the interface.

\section{Numerical Results}

Fig. 3 shows the relative Mach number in the reference case (without any mixing plane), in a blade-to-blade surface. The white lines indicate the three mixing plane locations and the purple line shows the iso-contour $M=1$. With the mixing plane located in (c), the subsonic zone between the leading edge and the shock wave is clearly cut. 


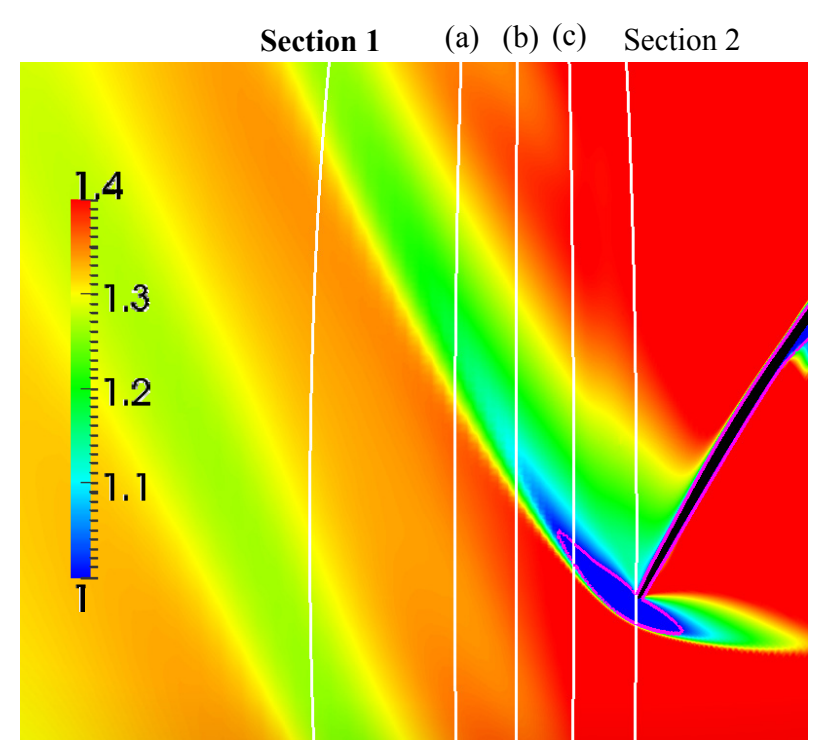

Fig. 3 Relative Mach number without mixing plane.

Fig. 4 shows that in the four configurations, the detachment distance of the shock remains constant, which tends to prove that the mixing plane gives a correct value of the averaged Mach number. Indeed, as explained in the following, the detachment distance only depends on the blade geometry and inlet Mach number. The white lines represent Mach iso-contours from 0.7 to 1.4 .

However, the shape of the subsonic zone is seriously affected. The more the mixing plane is located downstream, the less the shock waves can extend upstream. Thereby, according to the position of the mixing plane, the total pressure loss is under-estimated.

The change in stagnation pressure can be quantified with the value of the loss coefficient $K$ calculated as:

$$
K=1-\frac{\overline{P_{t 2}}}{\overline{P_{t 1}}}
$$

with $\overline{P_{t}}$, the momentum-averaged relative stagnation pressure integrated on the whole surface at Section 2 (located at the blade leading edge) and on the whole surface at Section 1 (located upstream) as:

$$
\bar{P}_{t}=\frac{\int_{S} P_{t} \cdot \rho V_{n} \cdot \mathrm{d} S}{\int_{S} \rho V_{n} \cdot \mathrm{d} S}
$$
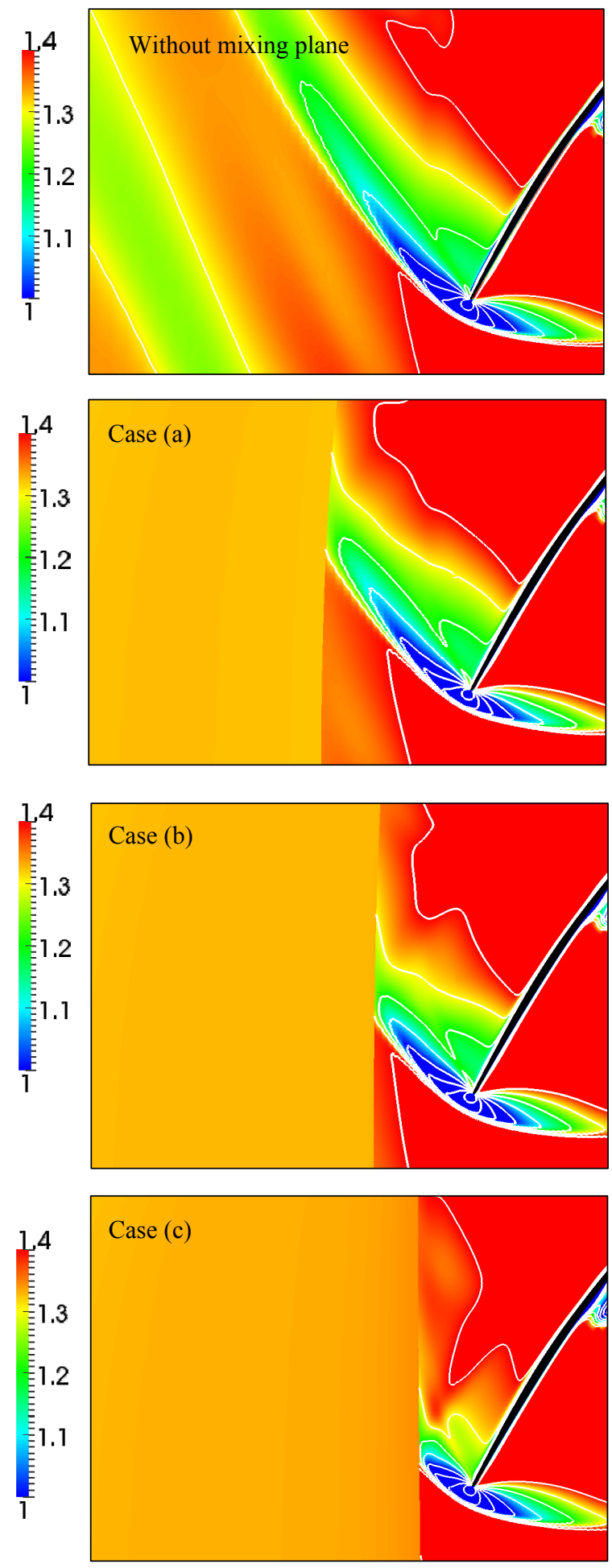

Fig. 4 Relative Mach number in the four test cases.

As is expected, the more the mixing plane is located downstream, the lower the losses are, and consequently, the more the massflow is over-estimated. Table 1 gives the difference between overall inlet massflow in cases 
Table 1 Performance shift to the reference configuration.

\begin{tabular}{lll}
\hline & $\Delta$ Massflow & $\Delta$ K/Kref \\
\hline Mixing plane located in (a) & $+0.04 \%$ & $-1.2 \%$ \\
Mixing plane located in (b) & $+0.35 \%$ & $-8.3 \%$ \\
Mixing plane located in (c) & $+0.80 \%$ & $-21.2 \%$ \\
\hline
\end{tabular}

(a), (b), (c) compared to the reference configuration without mixing plane.

The effect of the mixing plane can also be shown by the evolution of the entropy along a constant span height in the supersonic region (black arrow in the meridional view in Fig. 5). By preventing the shock wave to extend upstream, the mixing plane introduces a shift in the global level of entropy.

These data show that the result of the steady state simulation significantly depends on the mixing plane location both in terms of performance (massflow and loss) and flow topology. The objective of the following part is to propose a method which can be used to:

(1) forecast the location of the mixing plane minimizing the shift in performance;

(2) forecast the change in performance for a given mixing plane location.

\section{Analytical Description}

Let us consider a supersonic incoming flow compressor with subsonic axial velocity component. Depending on the inlet Mach number and on the back pressure level, two different regimes can exist:

- the unstarted regime, characterized by a detached, quasi-normal shock across the passage;

- the started regime, characterized by an attached oblique shock.

In case of a blunt leading edge, the shock cannot be strictly attached and a small subsonic area exists upstream the blade. The detachment distance of the shock is obviously smaller in the case of started regime than that of unstarted regime.

The model presented hereafter is only valid for a started regime. This is the first reason why the study takes place near the sonic blockage: the shock wave has to remain attached to the leading edge of the blades.

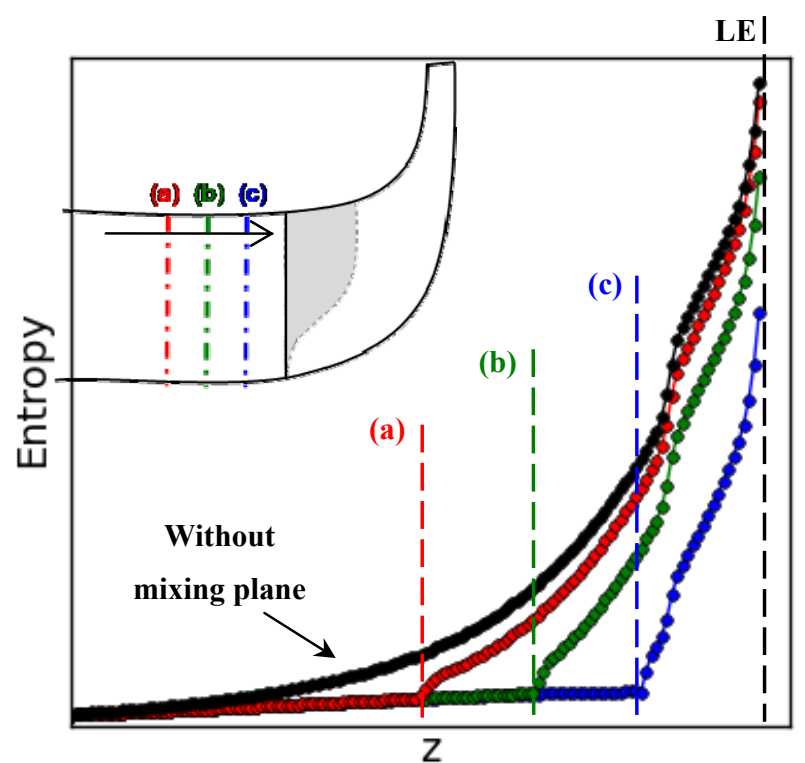

Fig. 5 Evolution of entropy along the rotation axis $z$, at a constant span height.

Moreover, the presence of splitter blades here is likely to influence the shock system at the leading edge of the main blades through potential effects. Thus, the back pressure has to be imposed very low.

The two inputs of this model are the upstream Mach number and the geometry of the blade leading edge. The detachment distance is calculated with Moeckel's method [8], which assumes that the detached shock has a hyperbolic shape (Fig. 6). The equation of the hyperbola is specified by its asymptote (of angle $\mu_{\infty}$ ) and the position of point $\mathrm{C}$ located on the sonic line $[\mathrm{BC}]$, which is supposed to be straight.

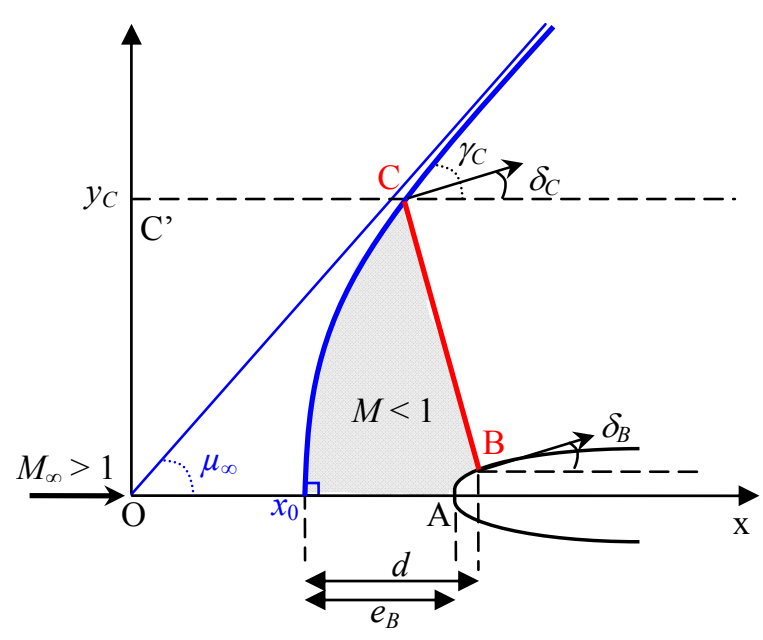

Fig. 6 Sketch of a detached shock. 
In order to apply Moeckel's model, the upstream flow is supposed to be two-dimensional, uniform and the profile is approximated by a symmetric shape. The thermodynamic shock relations for ideal gas are used. The maximum entropy rise, corresponding to a normal shock, is located on the streamline passing by the leading edge. This line and the sonic line are assumed to be straight. The available equations are:

- The equation of the hyperbola:

$$
y_{c}^{2}=\left(x_{c}^{2}-x_{0}^{2}\right) \operatorname{tg}^{2} \mu_{\infty}
$$

- The equation of the tangent at point $\mathrm{C}$ :

$$
\operatorname{tg} \gamma_{C}=\frac{x_{C}}{y_{C}} \operatorname{tg}^{2} \mu_{\infty}
$$

which, combined with Eq. (1), gives:

$$
x_{0}=y_{C} \frac{\sqrt{\operatorname{tg}^{2} \gamma_{C}-\operatorname{tg}^{2} \mu_{\infty}}}{\operatorname{tg}^{2} \mu_{\infty}}
$$

- The equation of the distance $e_{B}$ :

$$
\begin{aligned}
e_{B}=y_{C} \frac{\operatorname{tg} \gamma_{C}}{\operatorname{tg}^{2} \mu_{\infty}}+\left(y_{C}-y_{B}\right) \operatorname{tg} \delta_{C} \\
-y_{C} \frac{\sqrt{\operatorname{tg}^{2} \gamma_{C}-\operatorname{tg}^{2} \mu_{\infty}}}{\operatorname{tg}^{2} \mu_{\infty}}
\end{aligned}
$$

In order to calculate the ordinate of point $\mathrm{C}, y_{C}$, the continuity equation is written between the segment $\left[O C^{\prime}\right]$ upstream the shock wave and the sonic line $[\mathrm{BC}]$ :

$$
\rho_{\infty} V_{\infty} y_{C}=\rho_{C}^{*} a_{C}^{*} \overline{\mathrm{BC}}=\rho_{C}^{*} a_{C}^{*} \frac{y_{C}-y_{B}}{\cos \delta_{C}}
$$

which leads to:

$$
\frac{y_{C}}{y_{B}}=\frac{1}{1-\frac{\rho_{\infty} V_{\infty}}{\rho_{\infty}^{*} a_{\infty}^{*}} \frac{\rho_{\infty}^{*} a_{\infty}^{*}}{\rho_{C}^{*} a_{C}^{*}} \cos \delta_{C}}
$$

Since a shock wave is isenthalpic, we can write:

$$
\frac{\rho_{\infty} V_{\infty}}{\rho_{\infty}^{*} a_{\infty}^{*}}=\left(\frac{M_{\infty}\left(1+\frac{\gamma-1}{2} M_{\infty}^{2}\right)}{\frac{\gamma+1}{2}}\right)^{\frac{\gamma+1}{2(1-\gamma)}}
$$

$$
\frac{\rho_{\infty}^{*} a_{\infty}^{*}}{\rho_{C}^{*} a_{C}^{*}}=\frac{P_{t \infty}}{P_{t C}}
$$

It is important to notice that a choice is possible at that Eq. (8) in the way the stagnation pressure ratio is calculated. In the present case, a normal shock is considered at point $\mathrm{C}$ :

$$
\frac{\rho_{\infty}^{*} a_{\infty}^{*}}{\rho_{C}^{*} a_{C}^{*}}=\left[1+\frac{2 \gamma}{\gamma+1}\left(M_{\infty}^{2}-1\right)\right]^{\frac{-1}{\gamma-1}}\left[1-\frac{2}{\gamma+1}\left(1-\frac{1}{M_{\infty}^{2}}\right)\right]^{\frac{-\gamma}{\gamma-1}}
$$

It would also be thinkable to consider an oblique shock to evaluate the thermodynamic state along the sonic line. This is at the same time a source of uncertainty in this method and a degree of freedom for the user (playing on this ratio enables to fit CFD or measurements but it is rather difficult to give a formula which works for all types of blades).

The steps of the detachment distance calculation are thus:

- From the value of the inlet Mach number $M_{\infty}, \mu_{\infty}$ is calculated with:

$$
\mu_{\infty}=\arcsin \frac{1}{M_{\infty}}
$$

and the values of the deviation $\delta_{C}$ and shock angle $\gamma_{C}$ are deduced from the shock relations.

- Point $B$ which belongs to the profile is identified from its tangent $\delta_{B}$ which has to be equal to $\delta_{C}$.

- Eqs. (3), (4) and (6) are then used to calculate the detachment distance $d$ :

$$
d=e_{B}-\left(x_{B}-x_{A}\right)
$$

This procedure, initially thought for symmetric isolated profiles, leads to a geometric representation of the subsonic zone (in grey in Fig. 6), between the hyperbola and the sonic line. The part of the shock wave confining this subsonic region is responsible for most of the total pressure losses. Therefore, it is crucial to let it fully extend upstream the blades. The consequences of a mixing plane cutting the subsonic pocket are examined in the following. 


\section{Comparison between Analytical and Numerical Results}

The analytical method previously described for started regimes enables to draw the shape of the detached bow shock and the subsonic zone. Fig. 7 superimposes the analytical shape (in black) on the CFD results without any mixing plane (the yellow line is the iso-contour $M=1$ ).

Qualitatively, the subsonic area is well approximated. Assuming that the major part of the losses are due to the part of the shock confining the subsonic part, it is seen that the analytical calculation gives a pretty good evaluation of the minimal distance to be put between the mixing plane and the leading edge $\left(d_{\min }\right.$ in Fig. 7$)$.

However, two weaknesses should be pointed out.

Firstly, the model is very sensitive to the upstream Mach number, in particular in the low supersonic Mach numbers region (between $M_{\infty}=1.0$ and $M_{\infty}=1.1$ ). Fig. 8 shows the typical evolution of the detachment distance as a function of the upstream Mach number. Furthermore, when the upstream Mach number decreases, the shape of the subsonic pocket tends to become more elliptic. Fig. 9 shows that with $M_{\infty}=1.1$, the detachment distance and the orientation of the shock wave are still well estimated but Moeckel's method cannot reproduce the numerical result near the leading edge.

Secondly, the choice in the calculation of the loss across the shock leads to the thermodynamic state of the sonic line, because in the end, it directly drives the value of the detachment distance. Since Eq. (9) is non linear, the higher the upstream Mach number, the more important the way the loss is calculated becomes.

The main benefits of Moeckel's model are that only the geometry of the profile and the upstream Mach number are needed. As a consequence, this is a tool which can easily be employed in a pre-design phase. It should be underlined that the sonic blockage is the most "favorable" case because when the mass flow decreases, the compressor will pass from started

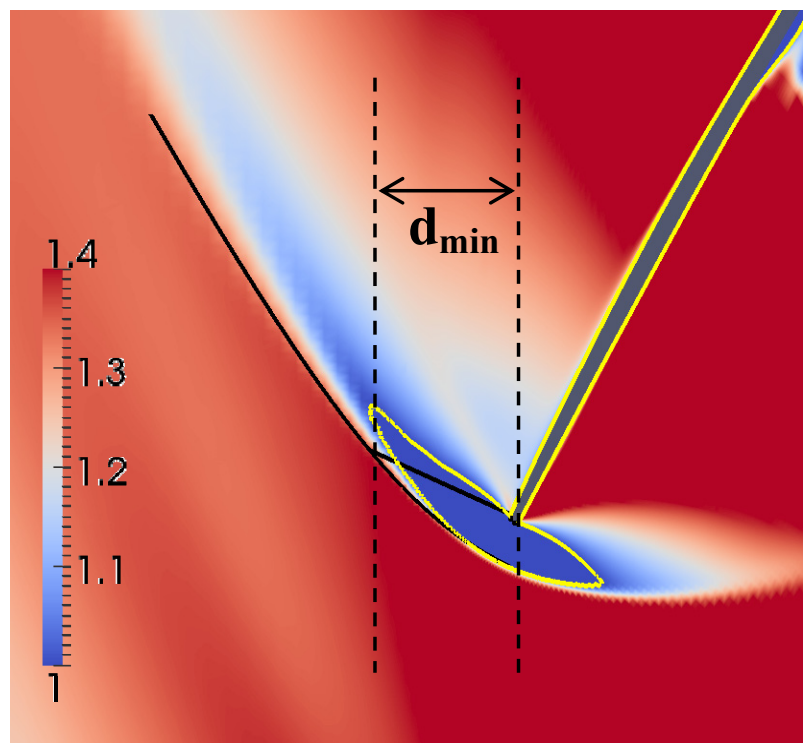

Fig. 7 Application of Moeckel's method with $M_{\infty} \approx 1.3$.

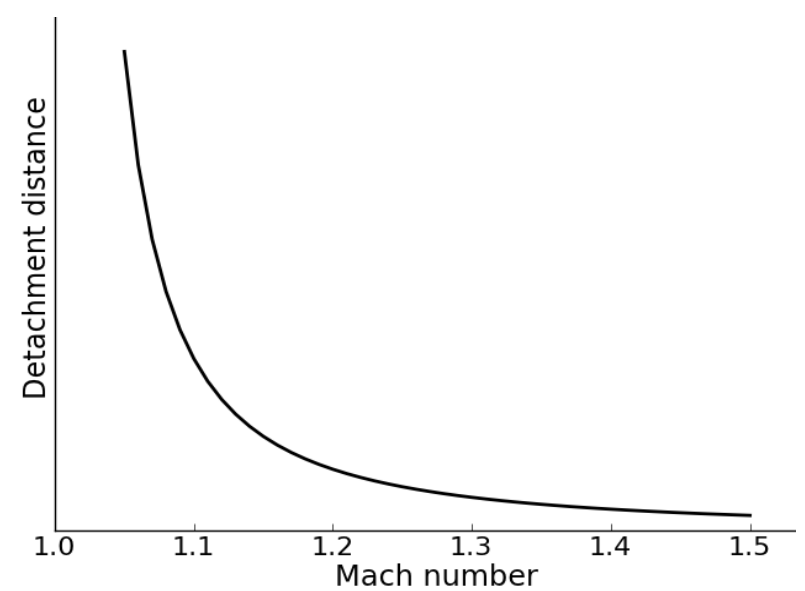

Fig. 8 Evolution of the detachment distance for a given profile.

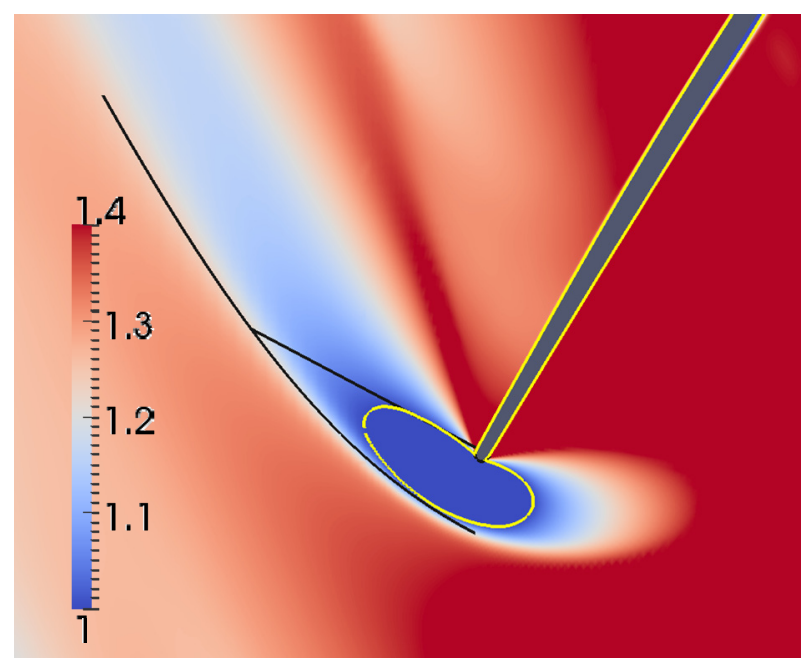

Fig. 9 Application of Moeckel's method with $M_{\infty} \approx 1.1$. 
regime to unstarted regime. Thus, the shock pattern moves upstream, so that the impact of the mixing plane can only become stronger.

\section{Shock Loss Prediction}

Although current blade design relies on numerical optimization, including transonic bladings [9], many analytical shock loss models exist, as described in Refs. $[10,11]$, for example. Bloch, Copenhaver and O'Brien also give an interesting approach based on Moeckel's method [12], adapted to unstarted regime.

Since the other objective of this paper is to analytically forecast the change in pressure loss for a given mixing plane location compared with the reference case (without any mixing plane), the shock loss associated to a started regime has to be evaluated. Fig. 10 shows the relative total pressure calculated in the reference case (without mixing plane): the low relative stagnation pressure zone at the leading edge plane corresponds to the projection of the strongest part of the shock on the leading edge plane in the mean flow direction. In order to be consistent with the numerical fields, only the part of the shock wave which is downstream the mixing plane location is kept and this portion of the upper branch of the hyperbola is projected in the plane of the leading edge (Fig. 11).

The losses are then calculated in the plane of the leading edge as if the fluid going through the bow shock was seeing a normal shock and the rest of the incoming flow remained unperturbed. The losses are evaluated with the coefficient $K$ defined as:

$$
K=1-\left(\frac{\xi}{t} \frac{P_{t 2}}{P_{t 1}}+\frac{t-\xi}{t}\right)
$$

where, $\xi$ is the length of the projected hyperbola, $t$ is the pitch and $P_{t 2} / P_{t 1}$ refers to the total pressure ratio across a normal shock (see Eq. (9)).

The value of $K$ is of course overestimated, since a part of the incoming fluid actually goes across an oblique shock and the bow shock extending upstream becomes rapidly evanescent. But it enables to evaluate the shock loss in the most unfavorable situation. Note

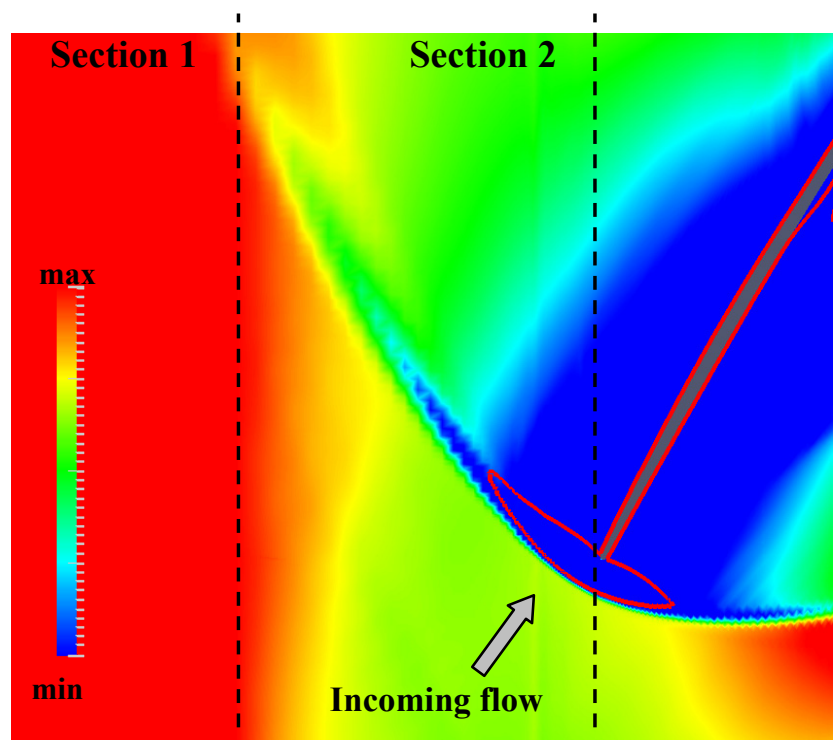

Fig. 10 Relative stagnation pressure in a blade-to-blade surface.

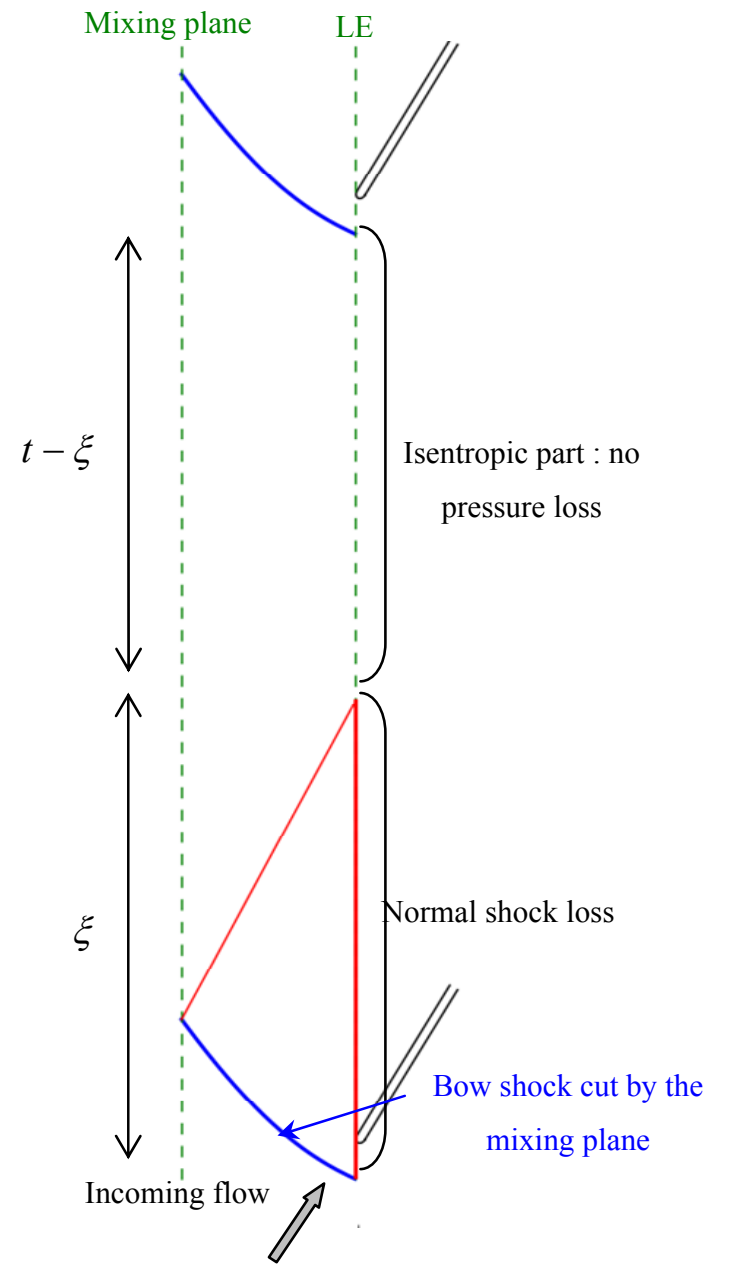

Fig. 11 Loss calculation for a given Mach number with a given mixing plane location. 
that in case of no mixing plane, the model is equivalent to considering a normal shock over the whole pitch.

This choice keeps the present tool simple, but it would be possible to make a more sophisticated model:

- First, by improving the shock wave approximation. A good example is given by Ottavy [13], by coupling Levine's method [14] to predict the unique incidence seen by the blade profile with Moeckel's detachment distance calculation.

- By discretizing the shock wave, so that the flow angle and the pressure loss change from a normal shock on the blade axis to an oblique shock and integrating the result on a pitch to the neighboring blade.

For a given profile, it is possible to plot the loss as a function of the upstream Mach number in the different configurations (Fig. 12). The case without mixing plane corresponds to the shock loss across a normal shock.

Depending on its location, the mixing plane has no more impact beyond a certain Mach number. For example, with a mixing plane located in Section (b) (green curve), it can be seen that beyond $M=1.46$, the mixing plane has no more influence. It means that if the upstream Mach number is higher than this value, the steady results can be considered reliable.

For a given mixing plane location, the discrepancy compared to the reference case firstly increases with the Mach number. Indeed, the length $\xi$ continuously increases but the pressure loss is increasing faster due to the shock wave. Then the upper branch of the hyperbola is more and more straightened up together with a decrease in the detachment distance until its projection covers the whole pitch. At that step, there is no more difference with or without a mixing plane and a steady RANS simulation can be considered as reliable (Fig. 13).

This analytical tool has been applied to the three mixing plane positions and to the reference case with no mixing plane. The input data are the circumferentially-averaged Mach numbers in Section (b) coming from the CFD with no mixing plane and the geometry of the front part of the blades.

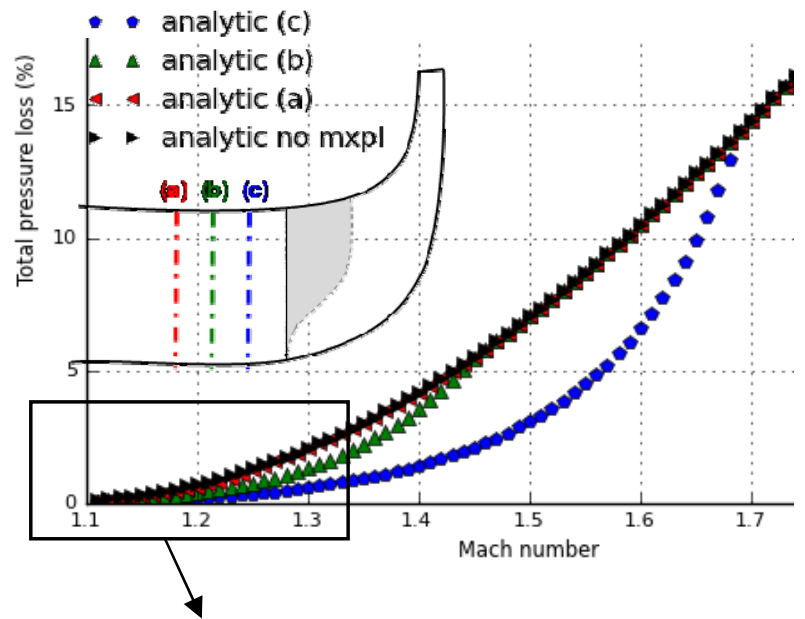

(a)

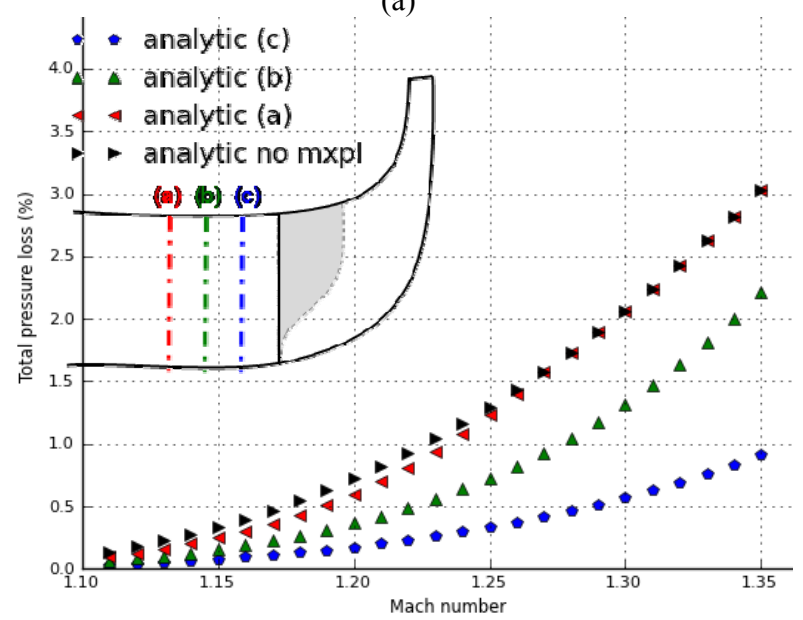

(b)

Fig. 12 (a) Evolution of $K$ for a given profile; and (b) zoom from Fig. 12a.

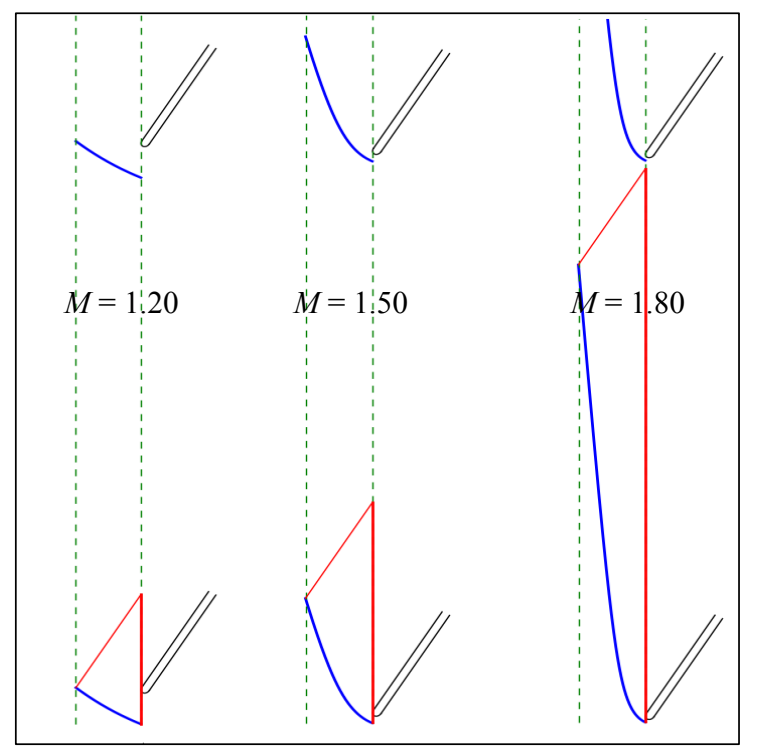

Fig. 13 Effect of the mixing plane on the bow shock for different upstream Mach numbers. 


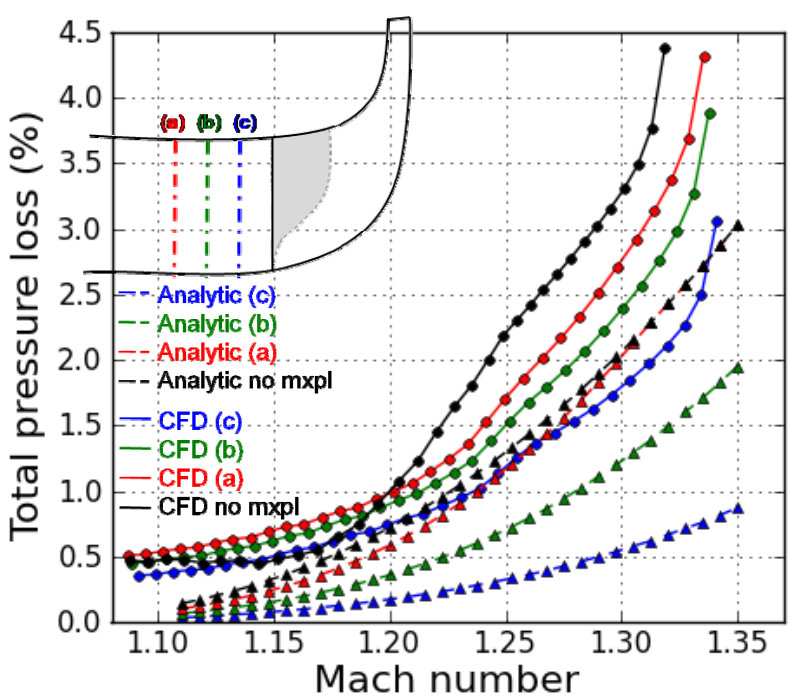

Fig. 14 Analytical and numerical loss as function of the upstream Mach number for different mixing plane locations.

Fig. 14 compares the analytical and numerical values obtained for the losses. Different section heights between $h_{1}$ and $h_{2}$ have been tested, so that both the profile and the Mach number were changing. The values of $K$ are plotted as a function of the upstream Mach number and are calculated as:

$$
K=1-\frac{\overline{P_{t 2}}}{\overline{P_{t 1}}}
$$

where,

$$
\overline{P_{t}}=\frac{\int_{0}^{2 \pi} P_{t} \cdot \rho V_{n} \cdot r \mathrm{~d} \theta}{\int_{0}^{2 \pi} \rho V_{n} \cdot r \mathrm{~d} \theta}
$$

In Fig. 14, the analytical evolution corresponding to case (a) is the same as the reference one for Mach numbers greater than 1.30 . This means that the major part of the hyperbola is contained downstream the mixing plane for the corresponding section heights. According to this criterion, any mixing plane should be located upstream Section (a) (Fig. 3) for the present impeller.

Nevertheless, despite the good qualitative agreement between analytical and numerical results in Fig. 7, we can observe quantitative discrepancies in the shock loss.
First of all, the simulation without mixing plane describes a different loss evolution around $M=1.20$ than those with a mixing plane. This is a well-known problem with mixing planes in general: the loss is radially redistributed.

The influence of the location of the mixing plane is clearly visible in the numerical curves. The slopes are not so far from the analytical ones. But there is a sort of offset between the numerical and the analytical results. It is probable that for the low Mach numbers, the shock loss is low compared to the viscous loss. To compare properly the two curves families, it would be necessary to take from the Navier-Stokes simulations only the shock loss, as done in Ref. [12] by subtracting the friction loss from measurements.

The discrepancies are also due to the strong hypotheses made in the analytical method, which consists in a two-dimensional approach and due to the choice made for the loss calculation. Real blade profiles are also often cambered and not symmetric in order to produce lift, which is not taken into account in the present model. And finally, the numerical loss at the highest Mach numbers (close to the section height $h_{2}$ ) is suddenly increasing, near the shroud boundary layer. It is likely that friction loss dominates shock loss in this area.

Thanks to this simple model, the order of magnitude of the under prediction of the losses due to the introduction of a mixing plane is easily evaluated. It has been tested that this approach gives acceptable results from inlet Mach number larger than 1.1. Once again, the major drawbacks of this method are that it gives wrong predictions for lower Mach numbers and that the shock formulas which are used in it are very sensitive. This is maybe one of the reasons why Bloch et al. [12] had to take into account an "effective" leading edge radius in their model dedicated to predict the shock loss through the lower branch of Moeckel's hyperbola, in supersonic compressor cascades operating in unstarted regime. Indeed, they increased the leading edge thickness until the analytical results 
matched the experimental ones. This reminds us of the difficulty of implementing a shock loss model that fits all types of profiles, under various operating conditions.

\section{Conclusion}

Steady state numerical simulations performed with a mixing plane approach show that the results, in terms of mass flow and losses, significantly depend on the mixing plane position. The operating point chosen here corresponds to the sonic blockage of the compressor but for near-surge points, it would be even more important.

The fact that this study takes place near the blockage enables to propose an analytical method in order to forecast this change in performance. The validity of this analytical method is checked by comparing its results with the numerical ones in the entry zone of a transonic compressor. Analytical and numerical results show good agreement.

This tool may be useful for transonic compressor design: first, to have an idea of the minimal distance that should be put between the mixing plane and the leading edge of the blades, and then to know how representatively the steady simulations can be expected.

\section{Acknowledgements}

We would like to thank Turbomeca which supported this study, together with ONERA which collaborated on the numerical simulation. This work was granted access to the HPC resources of CINES under the allocation 2013-2a6356.

\section{References}

[1] Lichtfuss, J. J., and Starken, H. 1974. "Supersonic Cascade Flow.” Progress in Aerospace Sciences 15: 37-149.
[2] Kantrowitz, A. 1946. The Supersonic Axial-Flow Compressor. NACA technical report.

[3] Chauvin, J. 1970. Supersonic Turbo-Jet Propulsion Systems and Components. AGARD report No. 120.

[4] Trébinjac, I., Ottavy, X., Rochuon, N., and Bulot, N. 2009. "On the Validity of Steady Calculations with Shock-Blade Row Interaction in Compressors." In Proceedings of the 9th International Symposium on Experimental and Computational Aerothermodynamics of Internal Flows, ISAIF9-062.

[5] Cambier, L., and Gazaix, M. 2002. "elsA: An Efficient Object-Oriented Solution to CFD Complexity." Presented at 2002 the 40th AIAA Aerospace Science Meeting and Exhibit, Reno, USA.

[6] Smith, B. R. 1995. "Prediction of Hypersonic Shock Wave Turbulent Boundary Layer Interactions with $k$ - $l$ Two-Equations Turbulence Model." Presented at 1995 the 33rd AIAA Aerospace Sciences Meeting and Exhibition, Reno, USA.

[7] Rochuon, N. 2007. "Analysis of the Three-Dimensional Unsteady Flow in a High Pressure Ratio Centrifugal Compressor." Ph.D. thesis, École centrale de Lyon.

[8] Moeckel, W. E. 1942. Approximate Method for Predicting Form and Location of Detached Shock Waves. NACA technical report.

[9] Burguburu, S., Toussaint, C., Bonhomme, C., and Leroy, G. 2004. "Numerical Optimization of Turbomachinery Bladings." Journal of Turbomachinery 126 (1): 91-100

[10] König, W. M., Hennecke, D. K., and Fottner, L. 1996. "Improved Blade Profile Loss and Deviation Angle Models for Advanced Transonic Compressor Bladings: Part II-A Model for Supersonic Flow." Journal of Turbomachinery 118 (1): 81-7.

[11] Schobeiri, M. T. 1997. "Advanced Compressor Loss Correlations, Part I: Theroretical Aspects." International Journal of Rotating Marchinery 3 (3): 163-77.

[12] Bloch, G. S., Copenhaver, W. W., and O'Brien, W. F. 1999. "A Shock Loss Model for Supersonic Compressor Cascades." Journal of Turbomachinery 121 (1): 28-35.

[13] Ottavy, X. 1999. "Laser Anemometry Measurements in an Axial Transonic Compressor. Analysis of the Unsteady Periodic Structures." Ph.D. thesis, École centrale de Lyon.

[14] Levine, P. 1956. The Two-Dimensional Inflow Conditions for a Supersonic Compressor with Curved Blades. WADC technical report 55-387. 